Design/ methods: 24 patients with PPHN were treated. Serum BNP levels were determined longitudinally. 3 patients were excluded because of the need for ECMO. In 6 patients a rebound of PPHN occurred after treatment was terminated. BNP levels were compared between infants with or without rebound PPHN ( $n=15)$.

Results: All PPHN infants had similar BNP levels at the start of initial NO. BNP levels decreased in both groups during NO treatment. In the infants who developed a rebound PPHN an increase was found in BNP shortly after cessation of NO treatment. This occurred well before the onset of clinical deterioration. BNP again decreased significantly during NO treatment $(p<0.05)$. Figure 1 provides the course of BNP.

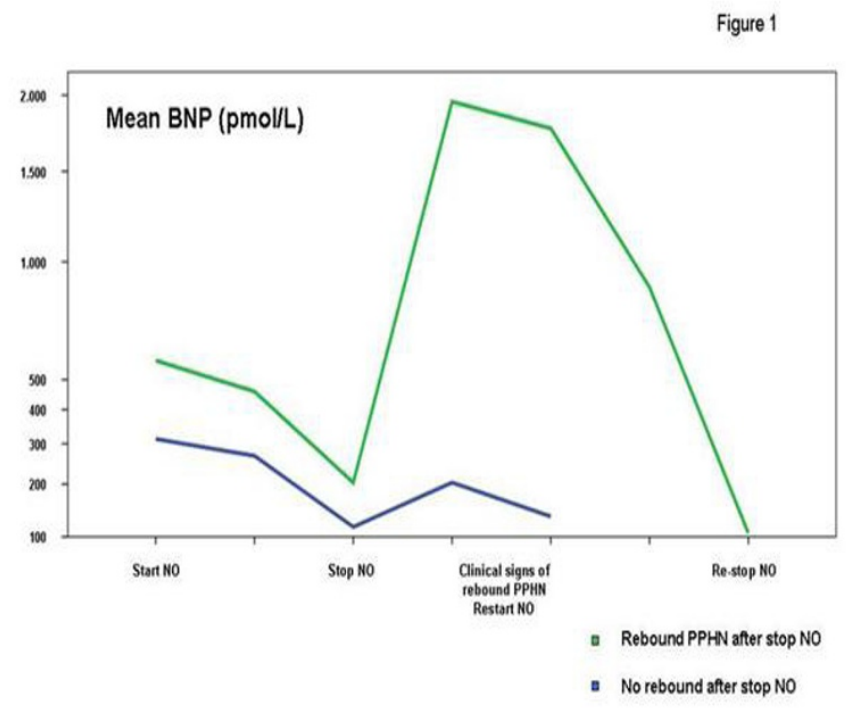

[Flgure 1]

Conclusion: BNP, as a biomarker of cardiac ventricular strain, proved to be useful in evaluating the course and treatment of PPHN and can serve as a predictor of rebound PPHN.
143

\section{SHOULD INFANTS WHO HAVE EARLY MAJOR SURGERY BE ENROLLED IN FOLLOW-UP CLINICS?}

K. Walker ${ }^{1,2}$, R. Halliday ${ }^{1}$, A.J. Holland ${ }^{2,3}$, D. Winlaw ${ }^{2,4}$, G. Sholler ${ }^{2,4}$, N. Badawi $i^{1,2,5,6}$

${ }^{1}$ Grace Centre for Newborn Care, The Children's Hospital at Westmead, 'The University of Sydney, ${ }^{3}$ Department of Surgery, ${ }^{4}$ Adolph Basser Cardiac Institute, The Children's Hospital at Westmead, ${ }^{5}$ Macquarie Foundation Professor of Cerebral

Palsy, Cerebral Palsy Institute, ${ }^{6}$ Notre Dame University, Sydney, NSW, Australia

Background: Survival following major surgery in early infancy is over $97 \%$ in Australia. This has focussed attention on the long term outcomes for these infants. Although there are many studies regarding developmental outcome of babies who have undergone cardiac surgery, the outcomes for babies who undergo other types of major surgery have been neglected. Unlike their preterm counterparts, infants who undergo major surgery are not routinely enrolled in newborn developmental follow-up clinics.

Objective: To compare the early developmental outcome of infants who underwent cardiac surgery with those who underwent major non-cardiac surgery and healthy controls.

Method: This prospective population-based study enrolled 784 infants between August 2006 and December 2008 from the three Children's Hospitals in New South Wales and their co-located maternity units. They were assessed at one year of age (corrected) using the five subscales (cognition, expressive and receptive language, gross and fine motor) of the Bayley Scales of Infants and Toddler Development (Version-III).

Results: Infants who underwent cardiac surgery scored significantly lower on all subscales than control infants $(p<0.001)$. Similarly, infants who underwent non-cardiac surgery also scored lower than the control infants on all subscales $(p<0.05)$. Infants who underwent cardiac surgery scored significantly lower than the infants who underwent non-cardiac surgery on four of the subscales $(p<$.05).Conclusion: These important early findings suggest that Infants who undergo noncardiac major surgery and cardiac surgery are at high risk of developmental impairment. Infants who undergo major surgery warrant systematic 
neurodevelopmental follow-up to determine their long term outcomes.

\section{4}

INCIDENCE, RISK FACTORS AND SEVERITY OF PULMONARY MORBIDITY IN INFANTS WITH CONGENITAL DIAPHRAGMATIC HERNIA BORN IN HIGH-VOLUME CENTRES IN EUROPE

L. van den Hout ${ }^{1}$, I. Reiss ${ }^{1}$, T. Schaible ${ }^{2}$,

I. Capolopu ${ }^{3}$, A. van Heijst ${ }^{4}$, M. Gorett Silva ${ }^{5}$, K. Allegaert ${ }^{6}$, A. Greenough ${ }^{7}$, D. Tibboel ${ }^{1}$

${ }^{1}$ Intensive Care and Paediatric Surgery, ErasmusMC-Sophia, Rotterdam, The Netherlands, ${ }^{2}$ Paediatric Intensive Care, Universitätsklinikum Mannheim, Mannheim, Germany, ${ }^{3}$ Paediatric Intensive Care, Bambino Gesù Children's Hospital, Rome, Italy, ${ }^{4}$ Neonatal Intensive Care, UMCN st Radboud, Nijmegen, The Netherlands, ${ }^{5}$ Paediatric Intensive Care, Hospital S Joao, Porto, Portugal, ${ }^{6}$ Neonatal Intensive Care, Universiteitsziekenhuis Gasthuisberg, Leuven, Belgium, ${ }^{7}$ Neonatal Intensive Care, King's College, London, UK

Background and aims: Newborns with congenital diaphragmatic hernia (CDH) may develop chronic lung disease (CLD). Our aim was to determine the incidence, severity and risk factors of CLD in infants with $\mathrm{CDH}$.

Methods: Data were collected about $426 \mathrm{CDH}$ patients born between 2005 and 2008 at 8 highvolume centres (> 10 admissions of infants with $\mathrm{CDH}$ per year) in Europe. The primary endpoint was CLD, defined as oxygen dependency at day 28. The severity of CLD (mild: $\mathrm{FiO}_{2} 0.21$; moderate: $\mathrm{FiO}_{2}$ 0.22-0.29; severe: $\mathrm{FiO}_{2} \geq 0.30$ or CPAP/ mechanical ventilation) was determined at day 56 or at discharge, whichever came first.

Results: At day 28, the mortality rate was $28 \%$ and the CLD incidence was $31 \%$. Of all patients with CLD, $31 \%$ had severe CLD, $15 \%$ moderate CLD and $54 \%$ had mild CLD. Compared to patients without CLD, patients with CLD had a lower lung-to-head ratio $(p<0.001)$, more often had an intrathoracic liver position $(p<0.001)$, required treatment for pulmonary hypertension ( $p<0.001)$, had a patch repair $(p<0.001)$, developed a pneumothorax $(p<$ $0.001)$ and required ECMO $(p<0.001)$. Independent risk factors for CLD were an intrathoracic liver position (OR 5.9, 95\% Cl 3.9-10.4) and a lower gestational age at birth (OR $0.86,95 \% \mathrm{Cl} 0.73$ 0.97). Patients with severe CLD more often had a pneumothorax $(p<0.001)$, patch repair $(p=0.035)$ and ECMO treatment $(p<0.001)$ than patients with mild to moderate CLD.

Conclusion: Pulmonary morbidity, which is a major problem in infants with $\mathrm{CDH}$, can be identified antenatally.

\section{5}

\section{CLINICAL AND GROWTH OUTCOMES FROM THE DINO (ㅁHA FOR THE IMPROVEMENT OF NEURODEVELOPMENTAL OUTCOME IN PRETERM INFANTS) TRIAL}

\author{
C.T. Collins ${ }^{1,2}$, B.J. Manly ${ }^{3,4}$, R.A. Gibson ${ }^{5}$, \\ T.R. Sullivan ${ }^{6}$, P. Ryan ${ }^{6}$, A.J. McPhee ${ }^{7}$, \\ P.G. Davis ${ }^{3,4}$, M. Makrides ${ }^{1,2}$, the DINO Steering \\ Committee
}

${ }^{1}$ Child Nutrition Research Centre, Women's and Children's Health Research Institute, North Adelaide, ${ }^{2}$ Discipline of Paediatrics, The University of Adelaide, Adelaide, SA, ${ }^{3}$ Neonatal Medicine,

Royal Women's Hospital, 'Paediatrics, The University of Melbourne, Melbourne, VIC, ${ }^{5}$ School of Agriculture, Food and Wine, ${ }^{6}$ Discipline of Public Health, The University of Adelaide, ${ }^{7}$ Neonatal Medicine, Children, Youth and Women's Health Service, Adelaide, SA, Australia

Background and aims: Higher-dose docosahexaenoic acid, DHA, $(\sim 1 \%$ total fats $)$ compared with standard-dose $(\sim 0.3 \%)$ in infants born < 33 weeks gestation improved the mental development of girls (JAMA, 2009). We report the effect on growth, allergic and respiratory symptoms.

Methods: Multicentre randomised controlled trial, stratified for sex, birth-weight $(<1250 \mathrm{~g}, \geq 1250 \mathrm{~g})$ and centre. Lactating women took tuna oil capsules (higher-dose DHA) or soy oil (standard); preterm infant formula with matching DHA composition was given if needed. Data collection included weight, length and head circumference weekly in-hospital and at term, 4, 12 and 18 months corrected age (CA); oxygen supplementation at 36 weeks post menstrual age (PMA) and parental reporting of medical diagnosis or drug treatment for atopic conditions.

Results: 657 infants were enrolled, 93.5\% completed 18-month follow-up. Significant benefits were seen in infants receiving higher-DHA, including greater length $(0.7 \mathrm{~cm})$ at 18 months $\mathrm{CA}(95 \% \mathrm{Cl} 0.1,1.4$ $\mathrm{cm}, \mathrm{P}=0.02)$; increases in length at 4 months $\mathrm{CA}$ 